\title{
Comparison Between Divergent and Convergent Synthesis Routes of Amine-Terminated Dendrimer Functionalized onto the Halloysite Nanotubes
}

ISSN: 2578-0255

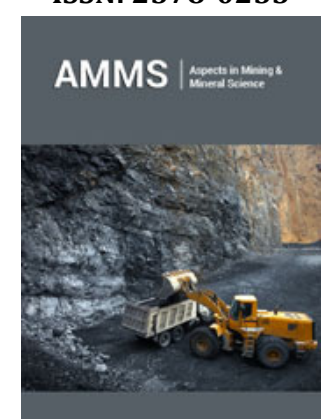

*Corresponding author: Somaye Akbari, Textile Engineering Department, Amirkabir University of Technology (Polytechnic Tehran), 424 Hafez Ave, Tehran, Iran

Submission: 侮July 02, 2019

Published: 㘹August 22, 2019

Volume 3 - Issue 3

How to cite this article: Mohammad Hassan Kanani-Jazi and Somaye Akbari. Comparison Between Divergent and Convergent Synthesis Routes of Amine-Terminated Dendrimer Functionalized onto the Halloysite Nanotubes. Aspects Min Miner Sci.3(3). AMMS.000564.2019.

DOI: 10.31031/AMMS.2019.03.000564

Copyright@ Somaye Akbari, This article is distributed under the terms of the Creative Commons Attribution 4.0 International License, which permits unrestricted use and redistribution provided that the original author and source are credited.

\section{Mohammad Hassan Kanani-Jazi and Somaye Akbari*}

Textile Engineering Department, Amirkabir University of Technology (Polytechnic Tehran), 424 Hafez Ave, Tehran, Iran

\begin{abstract}
This mini-review is about the enhancement of the surface activity and adsorption capacity of HNTs by amine-terminated dendritic polymers with two strategies: convergent and divergent synthetic routes. For this purpose, HNTs first was reacted through silanization reaction via 3-triethoxysilylpropylamine (APTES) to produce HNTs- $\mathrm{NH}_{2}$. In the divergent method, dendritic amino groups were added onto HNTs by the Michael addition of methyl acrylate (MA) to create half generation and then amidation reaction through ethylene diamine to produce full generation. This step was repeated to the dendritic structure grown onto HNTs step by step till define generation number. In the convergent synthesis route, two different strategies are possible. First, all hydroxyl groups were converted to carboxylic acid groups by dicarboxylic acid materials to produce HNTs- $\mathrm{COOH}$. Then, amine-terminated dendritic materials were grafted onto the carboxylic acid groups attached to HATs. In another method, amine-terminated dendritic materials were reacted with half generation (HNTs-MA) to create full generation. The result reveals that the convergent method has fewer steps than the divergent method, and its results lead to saving cost and energy.
\end{abstract}

Keywords: Dendrimer; Amine-Terminated group; Halloysite; Functionalization; Divergent; Convergent

Abbreviations: HNTs: Halloysite Nanotubes; MA: Methyl Acrylate

\section{Introduction}

Raw halloysite nanotubes (HNTs) typically are natural clay minerals with a unique hollow nanotubular structure includes two-layered aluminosilicate, adequate hydroxyl groups at its surface, and the gap space between its layers [1]. HNTs are a generously accessible natural nanomaterial and have chemical formula $\mathrm{Al}_{2} \mathrm{Si}_{2} \mathrm{O}_{5}(\mathrm{OH})_{4} \cdot 2 \mathrm{H}_{2} \mathrm{O}$ which formed from weathering and hydrothermal processes over millions of years [2]. HNTs have a variety of morphologies, such as spheroidal, platy, and tubular structure, the most well-known of which is the tubular form. It is abundant in many countries such as China, the United States, Brazil and France [3]. As a result, they are one of the cheapest existing adsorbents in many regions of the world. HNTs are made of octahedral gibbsite of $\mathrm{Al}-\mathrm{OH}$ groups on the inner surface and tetrahedral sheets of Si-O-Si groups on the outer surface [4]. The different chemical composition of the outer and inner surface of halloysite layers gives this nanomaterial novel features which do not exist in other clay minerals. These chemical compositions reveal a significant tendency to adsorb various guest compounds such as drugs [5,6], dyes heavy metal ions, and contaminant [7]. Hence, this clay mineral has received notable attention for many applications such as plastic composites, biomedical compounds, adsorbent wastewater due to its distinctive features of large specific surface area, large pore volume, high aspect ratio, mechanical strength, and low cost [8]. On the other hands, the presence of hydroxyl groups at the surface of HNTs allows this material to modify or functionalize with two or more functional groups [9]. Modification of clay minerals, especially halloysite and kaolinite to improve capacity or enhance the activity of these as adsorbent, is subject of numerous literatures. The first step typically involves washing off the powder to remove impurities such as iron, which often makes the active sites and then followed by activation with the coupling agent [10]. The common coupling agents are from the silane coupling agent group. Based on their literature, modified halloysite by organosilane 
seems to be a versatile host for loading several functional guests or functional groups [11]. Silane coupling agents are used attaching organic components to the inorganic base material. The activation sites are terminated by adding an organic component such as amines [12]. Most of the literatures have been emphasized that the amino functionalized halloysite reveals more reactive function comparing to hydroxyl function. It seems increasing amino function of halloysite by dendritic materials display additional characteristic to this mineral clay. In the past few decades, dendritic polymers as new and unique materials have attracted much attention due to their novel structure and features [13]. These materials are classified into four categories, including dendron, dendrigraft, hyperbranched polymers, and dendrimers. Dendritic polymers are a type of macromolecules characterized by their highly branched, a large number of end groups, globular shapes, compact shape, well-defined molecular weights and size, three-dimensional morphology, monodispersity, and free space between the branches for loading guest molecules [4,14-16]. Dendritic polymers adsorb guest molecules from aqueous solution in two ways: binding with the abundant functional end groups and encapsulating in the interior free space between the branches. Thus, functionalization halloysite by dendrimer provides a valid route for constructing highly efficient sorbent $[14,17]$. The present mini-review covers two different main methods for growing dendritic compounds onto the surface of HNTs by a dendritic polymer.

\section{Modification Method Via Divergent and Convergent}

\section{Dendritic Synthetic Route}

To introduce amine-terminated dendritic polymer on the surface of halloysite in either divergent or convergent synthetic routes first, aminosilane agents such as (3-Aminopropyl) triethoxysilane (APTES) were utilized to functionalize the halloysite in the process of silanization. In this case, amino groups are introduced on the surface of halloysite, which could act as the core for the synthesis of the dendritic structure on the halloysite via the divergent or convergent synthetic method.

\section{Modification in divergent synthetic method}

The first modification step to modify is changed all hydroxyl groups onto the surface of HNTs by amine groups via (3-Aminopropyl) triethoxysilane (APTES). In the divergent method, dendritic amine groups add onto HNTs by the Michael addition of methyl acrylate (MA). This step is repeated to the dendritic structure was grown onto HNTs-MA in half generation. Scheme 1 shows the first Michael addition of methyl acrylate to reach Generation G0.5 followed by amidation of the esters groups to terminate to Generation G1. By following the same Michael addition of methyl acrylate followed by amidation of the ester's groups, Generation G1.5 and G2 can be reached, which is also presented in Scheme 1. In the modification of HNTs, the reaction and termination could be continued to create generation $\mathrm{n}^{\text {th }}$. Several divergent method studies for modified HNTs were reported in the literature review. In one report, the amine-terminated functional groups were grown step by step onto the surface of HNTs to adsorption anionic dye molecules [17]. In another report, the modified HNTs by amine terminated dendritic materials via the divergent method (HNTs-G3) was used to adsorption cadmium and lead from aqueous solution [4]. Bahari et al. [18] were modified halloysite via divergent synthesis method and then Pd nanoparticles simultaneous immobilization onto the surface for catalyst application.

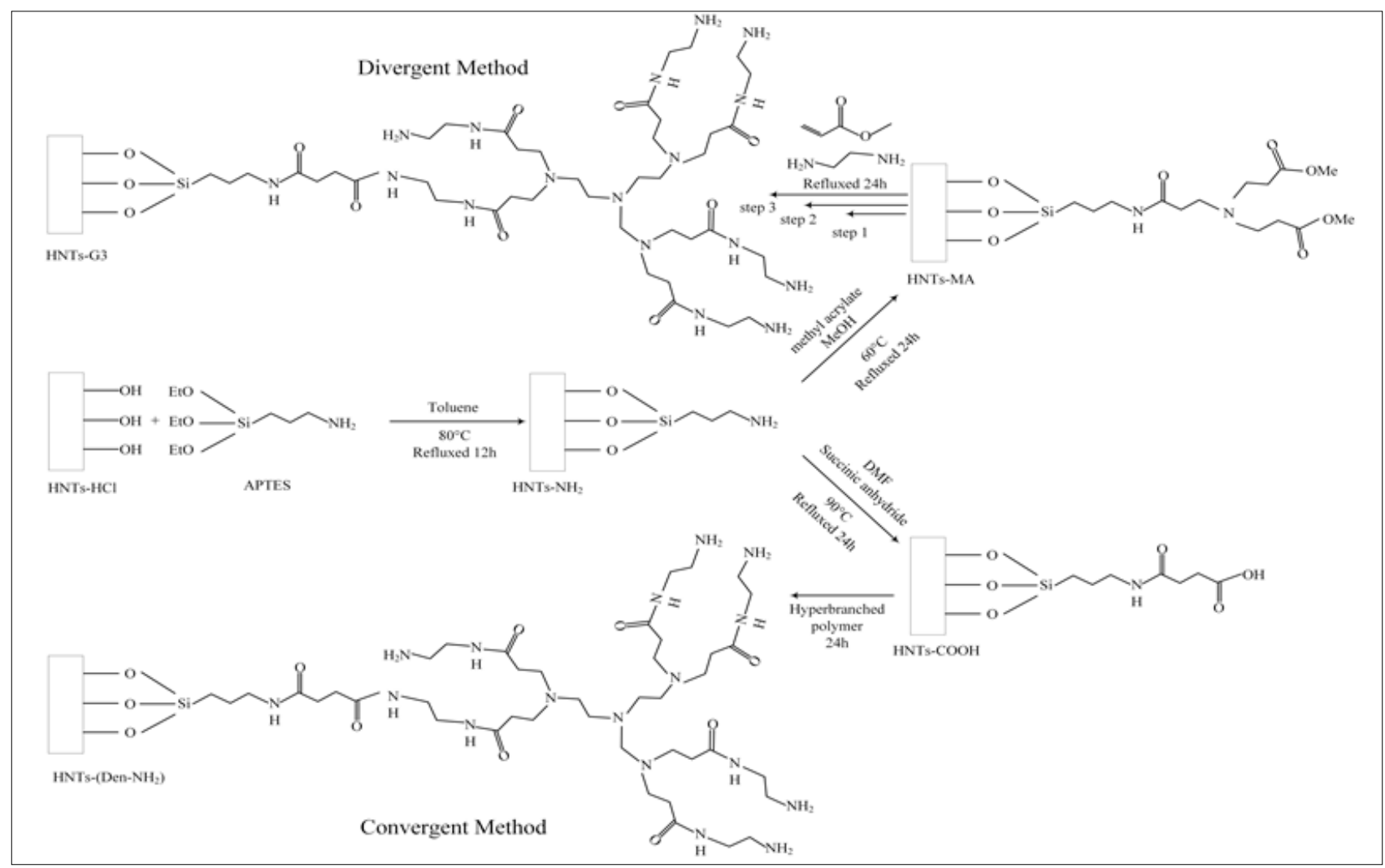

Scheme 1: Halloysite-amino terminated dendritic polymer via divergent and convergent synthesis routs. 


\section{Modification in convergent synthetic method}

Two different strategies could be applied in a convergent synthetic method:

a. Surface modification of halloysite nanotube (HNTs) via convergent method, compromising steps of: (1) in the first step, the HNTs were purified with hydrochloric acid, then the mixture was washed several times with distilled water to remove impurities until the $\mathrm{pH}$ value of the solution reached neutral and dried; (2) the amino groups were added onto surface of acidified HNTs by adding silane coupling agent solution ( $\left.\mathrm{HNTs}-\mathrm{NH}_{2}\right)$; (3) In the next step, the silane groups were replaced by carboxylic acid groups by adding succinic anhydride in DFM (HNTs-COOH). (4) Finally, the HNTs$\mathrm{COOH}$ was reacted by the dendritic polymer in methanol, ethanol or water. During this reaction, the dendritic polymer was placed onto the surface of HNTs (HNTs-Den- $\left(\mathrm{NH}_{2}\right)$.

b. The second strategy for placing dendritic compounds onto the surface of HNTs as follows: after purified HNTs and silane coupling, (1) the HNTs- $\mathrm{NH}_{2}$ were modified by Michael addition of methyl acrylate (MA) in methanol or ethanol (HNTs-MA), (4) Finally, the dendritic polymer attached onto surface of HNTs through reaction between HNTs-MA and dendritic polymer.

As shown in Scheme 1, the convergent method has fewer steps than the divergent method, and its results lead to saving cost and energy. Kurczewska et al. [19] were functionalized HNTs through convergent synthesis method for efficient drug delivery. The results indicate that the loading capacity of the drug after functionalization HNTs by amine terminated dendritic groups has increased significantly [19]. Polyamidoamine grafted onto the surface of HNTs via convergent synthesis method to efficient intracellular delivery of siRNA [20].

\section{Conclusion and Future Trends}

Halloysite nanotubes typically are natural clay minerals with a unique hollow nanotubular structure includes two-layered aluminosilicate. Hydroxyl groups are a presence at the surface of HNTs, and large pore volume makes them the best candidates for the modification with organic and inorganic materials to enhance loading capacity. Modified halloysite by organosilane seems to be a versatile host for loading several functional guests or functional groups. One of the most effective methods for modified halloysite is to use the dendritic compound. In this mini-review, the various methods of modified halloysite by amine dendritic polymer via convergent and divergent method were investigated. In the divergent method, dendritic amine groups add onto HNTs by the Michael addition of methyl acrylate (MA), and then the amineterminated were grown onto the surface step by step. In the convergent method, the silane groups were replaced by carboxylic groups, and then HNTs-COOH were grown by dendritic materials. In another convergent synthetic method, amine-terminated dendrimers were reacted by HNTs-MA. The result shows that the convergent method has fewer steps than the divergent method, and its results lead to saving cost and energy.

\section{References}

1. Joussein E, Petit S, Churchman J, Theng B, Righi D, et al. (2005) Halloysite clay minerals: A review. Clay Minerals 40(4): 383-426.

2. Abdullayev E, Abbasov VM, Lvov Y (2009) Halloysite clay nanotubes: Structural study and technological applications. J Petrochem Oil Ref 23: 234-246.

3. Ma W, Wu H, Higaki Y, Takahara A (2018) Halloysite nanotubes: Green nanomaterial for functional organic-inorganic nanohybrids. Chem Rec 18(7-8): 986-999.

4. Krawczyk M, Akbari S, Jeszka SM, Pajootan E, Fardb FS (2016) Application of dendrimer modified halloysite nanotubes as a new sorbent for ultrasound-assisted dispersive micro-solid phase extraction and sequential determination of cadmium and lead in water samples. J Anal At Spectrom 31(7): 1505-1514.

5. Massaro M, Campofelice A, Colletti CG, Lazzara G, Noto R, et al. (2018) Functionalized halloysite nanotubes: Efficient carrier systems for antifungine drugs. Appl Clay Sci 160: 186-192.

6. Zeng X, Sun Z, Wang H, Wang Q, Yang Y (2016) Supramolecular gel composites reinforced by using halloysite nanotubes loading with insitu formed $\mathrm{Fe}_{3} \mathrm{O}_{4}$ nanoparticles and used for dye adsorption. Compos Sci Technol 122: 149-154.

7. Wang Y, Zhang X, Wang Q, Zhang B, Liu J (2014) Continuous fixed bed adsorption of $\mathrm{Cu}(\mathrm{II})$ by halloysite nanotube-alginate hybrid beads: An experimental and modelling study. Water Sci Technol 70(2): 192-199.

8. Liu R, Fu K, Zhang B, Mei D, Zhang H, et al. (2012) Removal of methyl orange by modified halloysite nanotubes. J Dispers Sci Technol 33(5): 711-718.

9. Zargarian SS, Haddadi AV, Hematpour H (2015) Carboxylic acid functionalization of halloysite nanotubes for sustained release of diphenhydramine hydrochloride. J Nanoparticle Res 17:218.

10. Zhang AB, Pan L, Zhang HY, Liu ST, Ying Y, et al. (2012) Effects of acid treatment on the physico-chemical and pore characteristics of halloysite. Colloids Surfaces A: Physicochem Eng Asp 396: 182-188.

11. Zhang Y, Tang A, Yang H, Ouyang J (2016) Applications and interfaces of halloysite nanocomposites. Appl Clay Sci 119(1): 8-17.

12. Yuan P, Southon PD, Liu Z, Green M, Hook JM, et al. (2008) Functionalization of halloysite clay nanotubes by grafting with $\gamma$-aminopropyltriethoxysilane. J Phys Chem C 112(40): 15742-15751.

13. Malkoch M, Malmström E, Nyström AM (2012) Dendrimers: Properties and applications. Polym Sci A Compr Ref 10(6): 113-176.

14. Seiler M (2006) Hyperbranched polymers: Phase behavior and new applications in the field of chemical engineering. Fluid Phase Equilib 241(1-2): 155-174.

15. Tomalia DA, Fréchet JMJ (2002) Discovery of dendrimers and dendritic polymers: A brief historical perspective. J Polym Sci Part A Polym Chem 40(16): 2719-2728.

16. Li Y, Yang J, Huang C, Wang L, Wang J, et al. (2015) Dendrimerfunctionalized mesoporous silica as a reversed-phase/anion-exchange mixed-mode sorbent for solid phase extraction of acid drugs in human urine. J Chromatogr A 1392: 28-36.

17. Shahamati Fard F, Akbari S, Pajootan E, Arami M (2016) Enhanced acidic dye adsorption onto the dendrimer-based modified halloysite nanotubes. Desalin Water Treat 57(54): 26222-26239.

18. Bahri LN, Sadjadi S, Poater A (2018) Pd immobilized on dendrimer decorated halloysite clay: Computational and experimental study on the effect of dendrimer generation, Pd valance and incorporation of terminal functionality on the catalytic activity. J Colloid Interface Sci 531: 421-432. 
19. Kurczewska J, Cegłowski M, Messyasz B, Schroeder G (2018) Dendrimerfunctionalized halloysite nanotubes for effective drug delivery. Appl Clay Sci 153: 134-143.
20. Long Z, Wu YP, Gao HY, Li YF, He RR, et al. (2018) Functionalization of halloysite nanotubes via grafting of dendrimer for efficient intracellular delivery of siRNA. Bioconjug Chem 29(8): 2606-2618.

For possible submissions Click below: 